\title{
Tuberculosis Lymphadenitis in Northwest Ethiopia: Implications for Public Health
}

\author{
Fantahun Biadglegne $e^{1,2,3^{*}}$, Berhanu Anagaw ${ }^{4}$, Belay Anagaw ${ }^{5}$, Weghata Tesfaye ${ }^{5}$, Belay Tessema ${ }^{5}$, \\ Ulrich Sack $^{3,6,7}$, Arne C. Rodloff ${ }^{2}$ \\ ${ }^{1}$ Faculty of Medicine and Health Sciences, Bahir Dar University, Bahir Dar, Ethiopia \\ ${ }^{2}$ Institute of Medical Microbiology and Epidemiology of Infectious Diseases, University Hospital, \\ University of Leipzig, Leipzig, Germany \\ ${ }^{3}$ Institute of Clinical Immunology, University Hospital, University of Leipzig, Leipzig, Germany \\ ${ }^{4}$ Faculty of Science, Addis Ababa University, Addis Ababa, Ethiopia \\ ${ }^{5}$ College of Medicine and Health Sciences, University of Gondar, Gondar, Ethiopia \\ ${ }^{6}$ Fraunhofer Institute for Cell Therapy and Immunology, Leipzig, Germany \\ ${ }^{7}$ Translational Centre for Regenerative Medicine (TRM)-Leipzig, University of Leipzig, Leipzig, Germany \\ Email: *fantahun.degeneh@gmail.com
}

Received January 1, 2013; revised February 5, 2013; accepted February 24, 2013

\begin{abstract}
Objective: In Ethiopia where there are limited diagnostic facilities, the actual burden of tuberculosis (TB) lymphadenitis is not well known. Therefore, we conducted this study to determine the proportion of TB lymphadenitis (TBL) in childhood and adults in Northwest Ethiopia. Materials and Methods: A prospective cross sectional study was conducted from April to May 2012. Fine needle aspiration cytology (FNAC) for cytological diagnosis of TBL was used. The diagnosis of TBL was established when cytological features from lymph node aspirates are strongly suggestive of TB. Descriptive and multivariate analysis was done using SPSS version 16. Results: Out of 1070 patients attending the cytological diagnosis in the study sites 437 (41\%) were positive for TBL. Of the 437 registered TBL, 59 (13.5\%) were pediatric patients and $378(86.5 \%)$ were adults. There were more females than males with a male to female ratio of $0.8: 1$. The cervical region had the most common group of TBL with $321(73.2 \%)$ patients. Most of all these patients 314 $(75.3 \%)$, were matted with a majority of $(250 / 314)$ being in the cervical region. Individuals who had contact history with TB patients $(\mathrm{P}=0.046)$ were more likely to have TBL. Conclusions: In the studied region high prevalence of TBL was documented. Screening of TBL particularly for those who had contact with TB patients is recommended. Most patients in our study presented with matted lymph nodes, indicating that late arrival of patients to health institutions. Thus continuous and intensified public health strategies on health education and early referral system have to be done in order to link them to health institutions earlier than at present.
\end{abstract}

Keywords: FNAC; Epitheliod Cell Granulouma; Caseous Necrosis; TBL; Northwest Ethiopia

\section{Introduction}

Tuberculosis (TB) is one of the most serious public health challenges in Ethiopia. Indeed Ethiopia ranks 7 th among 22 countries world-wide with a high burden of TB. Both, pulmonary (PTB) and extra-pulmonary TB (EPTB) contribute to the problem. Thus, Ethiopia ranks 3rd in terms of the number of EPTB cases globally, most of which are TBL [1]. TBL is the most common form of EPTB [2]. Its epidemiology and diagnostic aspects vary according to the patients geographic origin and the burden of TB and human immunodeficiency virus (HIV) infection [3]. The proportion of EPTB among all TB cases varies from country to country. The proportion of

"Corresponding author.
EPTB among newly diagnosed TB patients, of which the majority of them is TBL, has been increasing for the last two years [4]. The World Health Organization (WHO) estimate that the proportion of EPTB among the total number of new TB cases is about $36.6 \%$ [5]. This contrasts with proportion of EPTB in other high TB burden countries in Asia, such as India (14.9\%), China (4\%), Indonesia $(2.5 \%)$, and in Africa such as South Africa $(17.5 \%)$, Nigeria (4.3\%) or Kenya (16.6\%) [6].

Children account for a substantial proportion of the global burden of TB [7]. Childhood TB remains neglected for various reasons, mainly the difficulty in diagnosis of PTB and the lack of scientific studies [8]. TBL was found in almost all the age groups, with a higher frequency at the $10-16$ years group (39.1\%); during this 
period of life, there is a maximum receptivity to the disease, with a low natural resistance of the body [9]. In India (9\%) of all children suffering from the disease [10], while in an African study it accounted $21 \%$ of pediatric cases admitted with tuberculosis [11]. In Ethiopia, childhood TB is still a major cause of morbidity and mortality [12]. Although previously considered a disease of childhood, TBL has also nowadays a peak onset age of 20 to 40 years [13]. The current level of TBL and its impact other than pulmonary tuberculosis is not clearly investigated in Ethiopia due to various reasons such as lack of expertise for diagnosis of TBL, lack of health care institutions and lack of reliable data particularly from rural areas. In this line; this study is aimed at investigating the proportion of TBL in childhood and adults in northwest Ethiopia.

\section{Materials and Methods}

\subsection{Study Design, Setting and Period}

This prospective cross sectional study was conducted in four health facilities, situated in North west Ethiopia with high notification rates of TBL that have facilities to carry out fine needle aspiration (FNA) and surgical biopsy (Felege Hiwot hospital and Gamby hospital in Bahir Dar, Gondar hospital in Gondar and Dessie hospital and Bikat diagnostic clinic in Dessie) were selected for the study that took place between April and May 2012.

\subsection{Patients and Procedures}

Patients who presented with chronic enlarged lymph nodes not responding to a two week course of broad spectrum antibiotics and were clinically diagnosed as isolated TBL [14] were our study subjects. Pyogenic abscesses were excluded from this study based on the clinical as well as cytomorphological features. The variables included in the study were age, sex, lymph node regions and whether the nodes were matted or discrete. The study was conducted after obtaining institutional ethical clearance from research and publication committee ethical review board of the Bahir Dar University, Bahir Dar, Ethiopia. As part of routine activity a pathologist collected fine needle aspirates of the affected nodes using a 22-gauge needle attached to a 10-cc syringe. The aspirate was macroscopically evaluated for caseation. Freshly purchased reagents were used and air dried smears per patient were prepared and stained with Wright stain for cytological diagnosis by experienced pathologists. TBL diagnosis by cytology was made based on presence of epitheloid cell granuloma with or without multinucleated giant cells and with or without caseous necrosis or degenerate caseous necrosis and/liquefied necrotic material with marked degenerating and viable inflammatory cell infiltration without epitheloid granu- loma [15]. Positive and negative slides were used as controls to measure the consistency of the staining technique. Data were entered, cleared, and analyzed using the SPSS statistical software package, Version 16. Descriptive data analysis was used to visualize differences within data and multivariate logistic regression was done to assess factors associated with TBL in terms of the odds ratio and its 95\% confidence interval (CI). Differences were considered significant when $\mathrm{p}$-value less than or equal to 0.05 .

\section{Results}

\subsection{Demographic Characteristics of Patients}

A total of 1070 subjects were enrolled from four study sites in northwest Ethiopia. Among these, 437 (41\%) were confirmed as TBL with cytological diagnosis. Of the 437 registered TBL patients, $59(13.5 \%)$ were pediatric patients ( $\leq 14$ years old) and $378(86.5 \%)$ were adults $(>14$ years old). There were more females $248(56.8 \%)$ than males $189(43.2 \%)$, with a male to female ratio of $0.8: 1$. In our findings more than half of the study subjects were farmers in occupation. Of these 24 (5.5\%) were pediatric patients. Table 1 shows the sociodemographic characteristics of the study subjects. Distribution of patients with TB lymphadenitis by age and sex are summarized in Table 2, where the most age group affected were 15 - 34 years, followed by $\leq 14$ years. The cervical region had the most common group of TBL with 321 patients (73.2\%), followed by axillary region with $64(15.3 \%)$, and the inguinal region $35(8.4 \%)$ patients. Most of all these patients $314(75.3 \%)$, were matted with a majority of $(250 / 314)$ being in the cervical region. Sixty six patients $(15.8 \%)$ presented with discrete adenopathy, in which $55(13.2 \%)$ found to be in the cervical region (Table 3$)$.

\subsection{Different Factors Associated with TBL}

Among 185 (42.7\%) of participants who had history of contact with TB patients, $32(7.4 \%)$ were pediatric age group. This study revealed that fever as constitutional symptoms was present in $50(11.5 \%)$ children and 325 (74.7\%) adult cases, weight loss in 49 (11.5\%) children and $316(74.4 \%)$ adults and cough was present in 18 (4.1\%) children and $127(29.1 \%)$ adult patients. The differences in TBL with respect to sex, fever, weight loss, night sweating and cough were not statistically significant between pediatric patients and adult patients. However, contact history with $\mathrm{TB}$ patients $(\mathrm{OR}=1.79,95 \%$ CI $1.02-3.12, \mathrm{P}=0.046)$ were significantly associated with TBL (Table 4).

\section{Discussion}

TBL is a common clinical problem in Ethiopia and is 
Table 1. Socio-demographic characteristics of the study participants (n = 437), Northwest Ethiopia, April to May 2012.

\begin{tabular}{|c|c|c|c|}
\hline \multirow{2}{*}{ Variables } & \multicolumn{2}{|c|}{ TBL cases } & \multirow[b]{2}{*}{ Total N (\%) } \\
\hline & Pediatric patients N (\%) & Adults patients N (\%) & \\
\hline Sex & $59(13.5)$ & $378(86.5)$ & 437 \\
\hline Male & $26(5.9)$ & $163(37.5)$ & $189(43.2)$ \\
\hline Female & $33(7.6)$ & $215(49.2)$ & $248(56.8)$ \\
\hline Educational level & $59(13.5)$ & $378(86.5)$ & 437 \\
\hline Illiterate & $27(6.2)$ & $200(46.2)$ & 227 ( 51.9) \\
\hline Above read and write & $31(7.2)$ & $179(16.9)$ & $210(48.1)$ \\
\hline Occupation & $59(13.5)$ & $378(86.5)$ & 437 \\
\hline Farmer & $24(5.5)$ & $211(48.4)$ & $235(53.9)$ \\
\hline Non farmer & $35(8.0)$ & $167(38.3)$ & $202(46.1)$ \\
\hline Marital status & $59(13.5)$ & $378(86.5)$ & 437 \\
\hline Single & $57(13.1)$ & $90(20.6)$ & $147(33.6)$ \\
\hline Married & $1(0.2)$ & $252(57.8)$ & $253(57.9)$ \\
\hline Divorced & $1(0.2)$ & $26(6)$ & $27(6.2)$ \\
\hline Widowed & $1(0.2)$ & $9(2.1)$ & $10(2.3)$ \\
\hline Religion & $59(13.5)$ & $378(86.5)$ & 437 \\
\hline Orthodox & $55(12.6)$ & $345(79.3)$ & $400(91.4)$ \\
\hline Muslim & $3(0.7)$ & $30(6.9)$ & $33(7.6)$ \\
\hline Catholic & $1(0.2)$ & $1(0.2)$ & $2(0.5)$ \\
\hline Protestant & $1(0.2)$ & $1(0.2)$ & $2(0.5)$ \\
\hline
\end{tabular}

Table 2. Distribution of patients with TB lymphadenitis by age and Sex, Northwest Ethiopia, April to May 2012.

\begin{tabular}{|c|c|c|c|}
\hline \multirow{2}{*}{ Age (years) } & \multicolumn{2}{|c|}{ TBL cases } & \multirow{2}{*}{ Total N (\%) } \\
\hline & Male N (\%) & Female N (\%) & \\
\hline$\leq 14^{*}$ & $26(5.9)$ & $33(7.6)$ & $59(13.5)$ \\
\hline $15-24$ & $52(11.9)$ & $70(16)$ & $122(27.9)$ \\
\hline $25-34$ & $54(12.4)$ & $82(18.8)$ & $136(31.1)$ \\
\hline $35-44$ & $23(5.3)$ & $33(7.6)$ & $56(12.8)$ \\
\hline $45-54$ & $20(4.6)$ & $16(3.7)$ & $36(8.2)$ \\
\hline $55-64$ & $9(2.1)$ & $11(2.5)$ & $20(4.6)$ \\
\hline$>64$ & $5(1.1)$ & $3(0.7)$ & $8(1.8)$ \\
\hline Total & $189(43.2)$ & $248(56.8)$ & $437(100)$ \\
\hline
\end{tabular}

*Pediatric age group.

Table 3. Clinical features of TBL patients by lymph node regions, Northwest Ethiopia, April to May 2012.

\begin{tabular}{ccccc}
\hline \multirow{2}{*}{ Lymph node regions } & \multicolumn{4}{c}{ Clinical features } \\
\cline { 2 - 5 } & Matted N (\%) & Discrete N (\%) & Other N (\%) & Total N (\%) \\
\hline Cervical & $250(57.2)$ & $55(13.2)$ & $16(3.8)$ & $321(73.2)$ \\
Axilla & $53(12.7)$ & $7(1.7)$ & $4(1)$ & $64(15.3)$ \\
Inguinal & $26(6.2)$ & $3(0.7)$ & $6(1.4)$ & $35(8.4)$ \\
Other & $5(1.2)$ & $1(0.2)$ & $11(2.6)$ & $17(4.1)$ \\
Total & $314(75.3)$ & $66(15.8)$ & $37(8.9)$ & $437(100)$ \\
\hline
\end{tabular}


Table 4. Patients' charactestics associated with TBL, Northwest Ethiopia, April to May 2012.

\begin{tabular}{|c|c|c|c|c|}
\hline \multirow{2}{*}{ Characteristics } & \multicolumn{2}{|c|}{ TBL patients } & \multirow{2}{*}{ OR $(95 \% \mathrm{CI})$} & \multirow{2}{*}{ P-value } \\
\hline & Pediatric patients, N (\%) & Adult patients N (\%) & & \\
\hline \multicolumn{5}{|l|}{ Sex } \\
\hline Male & $26(5.9)$ & $163(37.5)$ & $1.04(0.6-1.80)$ & 0.889 \\
\hline Female & $33(7.6)$ & $215(49.2)$ & 1 & \\
\hline \multicolumn{5}{|c|}{ History of contact with TB patients } \\
\hline Yes & $32(7.4)$ & $153(35.3)$ & $1.79(1.02-3.12)$ & 0.046 \\
\hline No & $26(6)$ & $222(51.3)$ & 1 & \\
\hline \multicolumn{5}{|l|}{ Fever } \\
\hline Yes & $50(11.5)$ & $325(74.7)$ & $0.87(0.40-1.88)$ & 0.689 \\
\hline No & $9(2.1)$ & $51(11.7)$ & 1 & \\
\hline \multicolumn{5}{|l|}{ Weight loss } \\
\hline Yes & $49(11.5)$ & $316(74.4)$ & $0.88(0.41-1.89)$ & 0.689 \\
\hline No & $9(2.1)$ & $51(12)$ & 1 & \\
\hline \multicolumn{5}{|l|}{ Night sweating } \\
\hline Yes & $48(11)$ & $330(75.9)$ & $0.68(0.32-1.44)$ & 0.301 \\
\hline No & $10(2.3)$ & $47(10.8)$ & 1 & \\
\hline \multicolumn{5}{|l|}{ Cough } \\
\hline Yes & $18(4.1)$ & $127(29.1)$ & $0.86(0.48-1.57)$ & 0.659 \\
\hline No & $41(9.4)$ & $250(57.3)$ & 1 & \\
\hline
\end{tabular}

difficult to diagnose in most rural health centers, because they do not provide culture and cytology/histology services. The diagnosis is usually made by employing conventional microscopy for acid-fast bacilli (AFB). This technique is simple and fast, but it lacks sensitivity [8]. The limited diagnostic capacity for TBL in the country remains a challenge to improving case detection rates. In this study all the TBL cases were newly diagnosed. Thus, the efforts to improve TB case detection in the country should be strengthened.

In countries with limited laboratory capacity and high tuberculous infections, the diagnosis of TBL was established when cytological features from lymph node aspirates are strongly suggestive of TB [15]. In this study the diagnosis of TBL was established using fine needle aspiration cytology (FNAC) method. It is a reliable and an excellent diagnostic tool $[4,16]$, even with negative $\mathrm{ZN}$ stains for AFB, the cytomorphological features on FNAC is sufficient for diagnosis of TBL [15]. The cytological criteria for diagnosis of TBL have been clearly defined [15]. In this study a total of 1070 subjects were enrolled from four study sites in northwest Ethiopia. Among these, FNAC revealed a positive tuberculous diagnosis in 437
$(41 \%)$. This proportion is higher than the proportions found in the previous studies conducted in northwest Ethiopia in Gondar (28.3\%) [17], Tigray and Amhara (39\%) [18], Southeast Ethiopia (19.5\%) [8] and the Afar region (28\%) [19]. However, similar descriptions were reported in southwest Ethiopia (40\%) [20] and (43\%) [21]. In contrast to our study Rahel et al. [4] also reported the occurrence of high proportion of TBL (78\%) in the country in 2009. Furthermore, other studies in different areas of the country also reported high level of TBL at (74.4\%) [22] and (69\%) [23]. According to WHO estimates for Ethiopia in 2011, EPTB accounts one third of the new cases of TB in the country [5]. However, it is not possible to compare the findings of our study to the national estimate because our study did not investigate all forms of EPTB. The reasons for the increased proportions of TBL in Ethiopia are unknown [4]. However, it has been suggested that delayed diagnosis of pulmonary tuberculosis, incomplete treatment of TB, HIV co-infection and other underlying diseases may have increased the TBL [24]. A study to identify risk factors for the increased proportion of TBL in the country is recommended. In our study $59(13.5 \%)$ were pediatric group 
aged less than or equal to 14 years and $378(86.5 \%)$ were adults. The most common age group affected in this study was 15 - 34 years, followed by pediatric age groups. This is in contrast to a study conducted in northwest Ethiopia in which the most age group affected was 35 44 followed by 45 years or older [21]. However, a similar result stating that TBL is primarily a disease of the young [16]. In the pediatric age group, Ramos et al. [8] reported high prevalence of EPTB, of which the majority of them is TBL (44.8\%). Marais et al. [25] also reported of the 35 children $27(77 \%)$ of them were positive for TBL. Narang et al. [26] reported (4.4\%) prevalence of TBL in children with a maximum prevalence was in the 5 - 9 years age group. The ratio of males to females in this study was $0.8: 1$, which is in contrast to the previous report by Mengstu et al. (1:1.4) [19] and Subrahmanyam (1:1.3) [27]. Studies have reported that females were more likely to be positive for TBL compared to males [28]. Similarly our finding also revealed that in both groups (pediatric and adults) high proportion of females were positive for TBL but the difference in prevalence in terms of gender was not stastically significant. In the patients described here, the cervical lymph nodes $(73.2 \%)$ were the prominent sites involved. The dominance of cervical lymph nodes has also been reported in other findings like Bezabih et al. (74.2\%) [16] and Narang et al $(73 \%)$ [26]. Cervical lymphadenitis is considered to have its origin in the lympho-hematogenous spread of organisms from a pulmonary focus via hilar and paratracheal lymph nodes ,but a minority of cases may originate from a primary focus in tonsils or tissues of the head and neck [26]. Three hundred and fourteen (75.3\%) were with matted nodes most often in the cervical regions 250 $(57.2 \%)$, followed by axillary regions 53 (12.7\%). This pattern suggests that the late arrival of patients in seeking medical care. It is fact that the health care seeking behavior could be low for TBL compared to that of pulmonary TB and patients with TBL may have had prior consultations with traditional healers and come late to health institutions [16]. Follow up and public health education focusing on raising awareness of the disease due to infection with TB bacteria, sources of infection, mode of transmission and preventive methods is very important for those TBL suspects. In our findings 66 (15.8\%) of them presented with discrete mobile lymph nodes indicating that the occurrence of early TBL (personal communication).

Previous studies showed that constitutional symptoms are important in supporting the diagnosis of TBL [21]. The most common systemic symptoms in both study subjects were fever $(86.2 \%)$, weight loss $(85.9 \%)$ and night sweating $(86.9 \%)$. Cough as signs and symptoms were found in $(33.3 \%)$ of the study subjects. Patel and Mehta observed weight loss in $77 \%$ and fever in $73 \%$ of the cases [29]. Jha et al. [30] observed weight loss in $(14 \%)$, Fever and cough in $(11 \%)$ of the cases. So in this respect our observation differs significantly from others. A contact history to TB patients was significantly associated with TBL indicating that there is transmission going on in the community although further study may be needed to identify transmission dynamics [21]. Studies have suggested that a predominance of M.tb in TBL in Ethiopia [31], although bovine transmission would be expected where raw milk consumption is common. The main limitations of our study were, delay to confirm with culture and PCR, lack of information about the HIV status, which might have determinant factor for the rise of TBL cases in developing countries [24] and another limitations for this study is the absence of CD4 count data. This may be important as some studies have shown that TBL occurs more often in individuals with cell counts less than 200 cells/ $\mu$ l [32], though other studies found no association between frequency of TBL and decreasing CD4 cell counts [33]. However, our study is one of the few that provides baseline information concerning the prevalence of TBL in children and adult groups in northwest Ethiopia, and this could be useful for planning appropriate TB control strategies, especially in the present study areas and in settings where TBL is common.

In conclusions, the results of this study revealed that the prevalence of TBL is high in the study area. Understanding the changes in the level of TBL will help in making a prompt diagnosis, leading to appropriate therapy. In areas where there is limited resources, introduction of easy, cost effective and time saving diagnostic method is important. In the study area FNAC was able to make the diagnosis of tuberculosis by demonstrating the cytomorphological feature of the mycobacteria in a high proportion of cases. This is therefore; patients with TBL should undergo FNAC to rule out TBL infection. Most patients in our study presented with matted lymph nodes, indicating that late arrival of patients to health institutions and hence continuous and intensified public health strategies on health education and early referral system have to be done in order to link them to health institutions earlier than at present. We also recommended screening of TBL cases that have contact history to TB patients.

\section{Acknowledgements}

The work presented in this paper was made possible by funding from the German Federal Ministry of Education and Research (BMBF, PtJ-Bio, 0315883). Parts of this study were carried out with financial support of Fraunhofer Institute for Cell Therapy and Immunology, Leipzig, Germany, the Institute of Medical Microbiology and Epidemiology of Infectious diseases and Clinical Immu- 
nology, University Hospital Leipzig, Germany, the German academic Exchange (DAAD) and University of Bahir Dar, Ethiopia. We express our deep appreciation to all pathologists, without their effort this paper would not have been materialized.

\section{REFERENCES}

[1] WHO, "Global Tuberculosis Control: a Short Update to the 2009 Report," World Health Organization, Geneva, 2009.

[2] A. Ilgazli, H. Boyaci, I. Basyigit and F. Yildiz, "Extrapulmonary Tuberculosis: Clinical and Epidemiologic Spectrum of 636 Cases," Archives of Medical Research, Vol. 35, No. 5, 2004, pp. 435-441. doi:10.1016/j.arcmed.2004.05.008

[3] S. E. Weis, P. K. Moonan, J. M. Pogoda, L. Turk, B. King, S. Freeman-Thompson and G. Burgess, "Tuberculosis in the Foreign-Born Population of Tarrant County, Texas by Immigration Status," American Journal of Respiratory and Critical Care Medicine, Vol. 164, No. 6, 2001, pp. 953-957.

[4] I. Raheal, Van Den H. Jan, W. Yimtubeznash, et al., "Is Tuberculosis Lymphadenitis Over-Diagnosed in Ethiopia? Comparative Performance of Diagnostic Tests for Mycobacterial Lymphadenitis in a High Burden Country," Scandinavian Journal of Infectious Diseases, Vol. 41, No. 6-7, 2009, pp. 462-468.

doi:10.1080/00365540902897697

[5] WHO, "Global Tuberculosis Control, 2011," World Health Organization, Geneva, 2011.

[6] WHO, "Global Tuberculosis Control, 2008," World Health Organization, Geneva, 2008.

[7] S. M. Graham, R. P. Gie, H. S. Schaaf, J. B. Coulter, M. A. Espinal and N. Beyers, "Childhood Tuberculosis: Clinical Research Needs," The International Journal of Tuberculosis and Lung Disease, Vol. 8, No. 5, 2004, pp. 648-657.

[8] J. M. Ramos, F. Reyes and A. Tesfamariam, "Childhood and Adult Tuberculosis in Rural Hospital in Southeast Ethiopia: A Ten Year Retrospective Study," BMC Public Health, Vol. 10, 2010, p. 215.

doi:10.1186/1471-2458-10-215

[9] P. Ileana, Polixenia, E. N. Veronica and S. Felicia, "Diagnosis of Tuberculosis Adenitis in Children," Jurnalulpediatrlui, 2007.

http://www.jurnalulpediatrului.ro/pages/detaliu.php articol=Pediatrics-III-7\&revista $=39-40$

[10] V. Seth, “Antituberculous Therapy in Children," Indian Journal of Pediatrics, Vol. 53, No. 2, 1986, pp. 174-198.

[11] H. M. Coovadia, "Tuberculosis in Children," In: H. M. Coovadia and S. R. Benatars, Eds., A Century of Tuberculosis, Oxfored University Press, Cape Town, 1991, pp. 91-100.

[12] S. Amsalu, Z. Hurrisa and S. Nuri, "Tuberculosis in Children, Northwest Ethiopia," Ethiopian Medical Journal, Vol. 45, No. 2, 2007, pp. 159-163.

[13] M. P. Golden and H. R. Vikram, "Extrapulmonary Tu- berculosis: An Overview," American Family Physician, Vol. 72, No. 9, 2005, pp. 1761-1768.

[14] Federal Ministry of Health, "Manual: National Tuerculosis Control Programme, Ethiopia," Addis Ababa, 2008.

[15] A. Sulaiman, S. Afshan, Tazeen, et al., "A Comparison of Fine Needle Aspiration Cytology with Ziehl Neelson Staining Technique in Diagnosis of Tuberculosis Lymphadenitis," A Comprehensive Cytopathology, Vol. 16, No. 4, 2010, pp. 707-709.

[16] M. Bezabih, D. W. Mariam and S. G. Selassie, "Fine Needle Aspiration of Suspected Tuberculous Lymphadenitis," Cytopathology, Vol. 13, No. 5, 2002, pp. 284-290. doi:10.1046/j.1365-2303.2002.00418.x

[17] T. Belay, M. Abebe, B. Assegedech, D. Reissig, F. Emmrich and U. Sack, "Treatment Outcome of Tuberculosis Patients at Gondar University Teaching Hospital, Northwest Ethiopia. A Five-Year Retrospective Study," BMC Public Health, Vol. 9, 2009, p. 371. doi:10.1186/1471-2458-9-371

[18] MOH, "Tuberculosis, Leprosy and TB/HIV Prevention and Control Program Case Finding and Treatment out Reports," Ministry of Health of Ethiopia, Addis Ababa, 2002.

[19] L. Mengistu, A. Gobena, M. Gezahegne, M. Girmay, B. Gunner and A. Fekadu, "Knowledge of Cervical Tuberculosis Lymphadenitis and Its Treatment in Pastoral Communities of the Afar Region, Ethiopia," BMC Public Health, Vol. 11, 2011, p.157. doi:10.1186/1471-2458-11-157

[20] M. A. Yassin, J. O. Olobo, D. Kidane, Y. Negesse, E. Shimeles and A. Tadesse, "Diagnosis of Tuberculous Lymphadenitis in Butajira, Rural Ethiopia," Scandinavian Journal of Infectious Disease, Vol. 35, No. 4, 2003, pp. 240-243. doi:10.1080/00365540310004027

[21] A. Gemeda, D. Amare, A. Ludwig, et al., "Tuberculosis Lymphadenitis in South West Ethiopia: A Community Based Cross-Sectional Study," BMC Public Health, Vol. 12, 2012, p. 504. doi:10.1186/1471-2458-12-504

[22] J. M. Ramos, F. Reyes, R. Facin and A. Tesfamariam, "Surgical Lymph Node Biopsies in a Rural Ethiopian Hospital: Histopathologic Diagnoses and Clinical Characteristics," Ethiopian Medical Journal, Vol. 46, No. 2, 2008, pp. 173-178.

[23] D. Beyene, S. Ashenafi, L. Yamuah, A. Aseffa, et al., "Diagnosis of Tuberculous Lymphadenitis in Ethiopia: Correlation with Culture, Histology and HIV Status," The International Journal of Tuberculosis and Lung Disease, Vol. 12, No. 9, 2008, pp. 1030-1036.

[24] C. Bem, P. S. Patil, H. Bharucha, K. Namaambo and N. Luo, "Importance of Human Immuno Deficiency VirusAssociated Lymphadenopathy and Tuberculous Lymphadenitis in Patients Undergoing Lymph Node Biopsy in Zambia," British Journal of Surgery, Vol. 83, No. 1, 1996, pp. 75-78. doi: $10.1002 /$ bjs. 1800830124

[25] B. J. Marais, C. A. Wright, H. S. Schaaf, R. P. Gie, A. C. Hesseling, D. A. Enarson and N. Beyers, "Tuberculous Lymphadenitis as a Cause of Persistent Cervical Lymphadenopathy in Children from a Tuberculosis-Endemic Area," The Pediatric Infectious Disease Journal, Vol. 25, 
No. 2, 2006, pp.142-146. doi:10.1097/01.inf.0000199259.04970.d1

[26] P. Narang, R. Narang, R. Narang, D. K. Mendiratta, S. M. Sharma and N. K. Tyagi, "Prevalence of Tuberculous Lymphadenitis in Children in Wardha District, Maharashtra State, India," The International Journal of Tuberculosis and Lung Disease, Vol. 9, No. 2, 2005, pp. 188-194.

[27] M. Subrahmanyam, "Role of Surgery and Chemotherapy for Peripheral Lymph Node Tuberculosis," British Journal of Surgery, Vol. 80, No. 12, 1993, pp. 1547-1548. doi:10.1002/bjs. 1800801218

[28] J. F. Garcia-Rodriguez, H. Alvarez-Diaz, M. V. LorenzoGarcia, A. Marino-Callejo, A. Fernandez-Rial and P. Sesma-Sanchez, "Extrapulmonary Tuberculosis: Epidemiology and Risk Factors," Enfermedades Infecciosas y Microbiología Clínica, Vol. 29, No. 7, 2011, pp. 502-509. doi:10.1016/j.eimc.2011.03.005

[29] R. V. Patel and R. T. Mehta, "Short Term Chemotherapy in Tuberculous Lymphadenitis," Indian Journal Surgery, Vol. 49, 1987, pp. 336-341.

[30] B. C. Jha, A. Dass, N. M. Nagarkar, R. Gupta and S. Singhal, "Cervical Tuberculous Lymphadenopathy: Chang- ing Clinical Pattern and Concepts in Management," Postgraduate Medical Journal, Vol. 77, No. 9, 2001, pp. 185-187. doi:10.1136/pmj.77.905.185

[31] D. Beyene, I. Bergval, E. Hailu, S. Ashenafi, L. Yamuah, A. Aseffa, H. G. Wiker, H. Engers, P. Klatser and L. Sviland, "Identification and Genotyping of the Etiological Agent of Tuberculous Lymphadenitis in Ethiopia," The Journal of Infection in Developing Countries, Vol. 3, No. 6, 2009, pp. 412-419. doi: $10.3855 /$ iidc. 411

[32] R. W. Shafer, D. S. Kim, J. P. Weiss and J. M. Quale, "Extrapulmonary Tuberculosis in Patients with Human Immunodeficiency Virus Infection," Medicine (Baltimore), Vol. 70, No. 6, 1991, pp. 384-397. doi:10.1097/00005792-199111000-00004

[33] C. Perronne, A. Ghoubontni, C. Leport, D. Salmon-Ceron, F. Bricaire and J. L. Vilde, "Should Pulmonary Tuberculosis Be an AIDS-Defining Diagnosis in Patients Infected with HIV?" Tuber Lung Disease, Vol. 73, No. 1, 1992, pp. 39-44. doi:10.1016/0962-8479(92)90078-X 